\title{
OTIMIZAÇÃO DA ALOCAÇÃO TEMPORAL DE RECURSOS PARA COMBATE A EPIDEMIAS COM TRANSMISSÃO SAZONAL ATRAVÉS DE MÉTODOS DE BARREIRA
}

\author{
Arlindo R. Galvão Filho*, Roberto KaWakami Harrop Galvão*, Takashi Yoneyama* \\ * Divisão de Engenharia Eletrônica \\ Instituto Tecnológico de Aeronáutica \\ São José dos Campos, São Paulo, Brasil
}

Emails: arlindo@ita.br, kawakami@ita.br, takashi@ita.br

\begin{abstract}
The application of mathematical models to epidemiology has contributed to the understanding of the dynamics of various infectious diseases, and possible strategies for more effective control. The classical approach uses differential equations to describe, in a quantitative way, the spread of disease within a given population. An example is the widely used Susceptible-Infected-Recovered compartmental model. This model has highlighting for obtaining optimal control policies involving seasonal dynamics, limited resources, among others. Given that the analysis of the time allocation of the control action is a decisive factor in making decisions on resources application this paper proposes a search for optimal control with limited resources considering seasonal dynamics of contagion and a time constant associated with effectiveness of the campaign. To illustrate such a scenario the proposed model was based on the dynamics of the seasonal influenza.
\end{abstract}

Keywords - Mathematical Epidemiology, SIR, Optimal Control, Seasonal Influenza.

Resumo - A aplicação de modelos matemáticos à epidemiologia tem contribuído para o entendimento da dinâmica de várias doenças infecciosas, bem como possíveis estratégias de controle mais eficazes. A abordagem clássica utiliza equações diferenciais para descrever, de forma quantitativa, a propagação de doenças dentro de uma determinada população. Um exemplo amplamente utilizado é o modelo compartimental SuscetívelInfectado- Recuperado. Tal modelo vem se destacando para obtenção de políticas de controle ótimo envolvendo dinâmicas sazonais, recursos limitados entre outras. Tendo em vista que a análise do tempo de alocação da ação de controle é um fator decisivo na tomada de decisões em aplicações de recursos este trabalho propõe uma busca pelo controle ótimo com recursos limitados considerando uma dinâmica de contágio sazonal e uma constante de tempo associada à efetivação da campanha. Para ilustrar tal cenário o modelo proposto foi baseado na dinâmica da influenza sazonal.

Palavras-chave- Epidemiologia Matemática, SIR, Controle Ótimo, Influenza Sazonal.

\section{Introdução}

Epidemiologia é uma importante área da ciência que envolve o estudo de fenômenos associados à saúde das populações humanas, bem como possíveis fatores de risco que possam condicionar doenças (Filho and Rouquayrol, 2006). Dentro de tal área, existe um importante campo de investigação chamado epidemiologia matemática, que se caracteriza pelo uso de modelos matemáticos para o desenvolvimento de estratégias de controle e prevenção de epidemias (Anderson and May, 1992).

Um dos modelos mais utilizados considera que a população possa ser dividida em três classes: Suscetíveis, Infectados e Recuperados (SIR). A dinâmica da epidemia é então representada por um conjunto de equações diferenciais determinísticas que descrevem a evolução temporal do número de indivíduos em cada uma das classes (Kermack and Mckendrick, 1927; Morton and Wickwire, 1974; Behncke, 2000). O SIR tem proporcionado bons resultados para investigação de aspectos gerais relacionado à propagação de diversas doenças infecciosas (Grenfell et al., 2002; Lu et al., 2002; Yoshida and Hara, 2007).

Uma doença bastante recorrente na população humana é a Influenza comum, também chamada de influenza sazonal. A influenza ocorre por todo o ano, mas verifica-se uma maior frequência nos meses mais frios do ano. No Brasil, segundo o Ministério da Saúde, foram registradas 750 mil internações por influenza no ano de 2011 (Ministério da Saúde, 2012). Segundo a Organização Mundial de Saúde, a influenza sazonal resulta em cerca de 250 a 500 mil mortes no mundo (World Health Organization, 2009).

O vírus da influenza é transmitido facilmente de uma pessoa infectada para outra por meio de perdigotos, ou seja gotículas disseminadas através de espirro, tosse, ou até mesmo contato das mãos com superfícies contaminadas.

A política mais comum e eficaz para a prevenção da epidemia é a vacina, que imuniza o indivíduo através do virus inativo. Porém as políticas adotadas atualmente também destinam recursos no intuito de prevenir a transmissão da doença pelo uso de métodos de barreiras entre o doente e seus contactos. Pode-se exemplificar tais métodos pelo uso de máscaras ou até mesmo pela adoção de medidas de higiene respiratória, como cobrir a boca ou o nariz ao tossir ou espirrar.

Existem diversos trabalhos propondo adaptações do SIR para representar a dinâmica da influenza e encontrar um controle ótimo para 
alocação dos recursos, considerando sazonalidade (Prosper and Saucedo, 2011), recursos limitados (Hansen and Day, 2011), campanha de saúde (Behncke, 2000), entre outros aspectos.

Os métodos de barreira são, na maioria dos casos, fornecidos para a população através de campanhas publicitárias de um modo geral. Isso agrega uma desvantagem ao método uma vez que leva-se um tempo para que o indivíduo tenha acesso ao método e o torne efetivo em sua rotina. Desta forma não é uma tarefa trivial saber qual o momento mais oportuno para alocar os recursos, levando em consideração o tempo gasto pela assimilação dos métodos de barreira e a sazonalidade da influenza.

Neste contexto, a proposta deste trabalho consiste em explorar o uso do SIR para otimizar a alocação temporal de recursos limitados para combate da influenza sazonal com métodos de barreira.

Este artigo está organizado da seguinte forma. Na Seção 2 é apresentado o modelo SIR. Na Seção 3 , descrevem-se o material e os métodos utilizados. Os resultados são discutidos na Seção 4. A Seção 5 traz as conclusões do trabalho.

\section{O modelo SIR}

O modelo SIR é constituído por um sistema de equações diferenciais não-lineares a tempo contínuo. Este modelo divide a população em três classes: suscetíveis (S), infectados (I) e recuperados (R). Os suscetíveis são pessoas que nunca foram infectadas e estão sujeitas a contágio através do contato com pessoas infectadas. Os infectados são pessoas que possuem a doença considerada e podem transmiti-la para pessoas suscetíveis. Por fim, os recuperados são pessoas que já foram infectadas, mas estão curadas e, por hipótese, adquiriram imunidade (Giancotti et al., 2010; Galvão Filho et al., 2011).

Por simplicidade, considera-se neste trabalho que o tamanho da população se mantém constante ao longo do tempo, ou seja, assume-se que as taxas de natalidade $(\mu)$ e mortalidade $(v)$ são iguais. Também foi considerado que não há transmissão vertical da doença, ou seja somente nascerão indivíduos susceptíveis. Deste modo o modelo SIR é dado por

$$
\begin{aligned}
d S(t) / d t & =\mu N-\mu S(t)-\beta \frac{S(t) I(t)}{N} \\
d I(t) / d t & =\beta \frac{S(t) I(t)}{N}-\gamma I(t)-\mu I(t) \\
d R(t) / d t & =\gamma I(t)-\mu R(t)
\end{aligned}
$$

em que $S(t)+I(t)+R(t)=N, \beta$ é a taxa de infecção e $\gamma$ é a taxa de recuperação.

\subsection{Dinâmica sazonal do contágio}

Segundo o Ministério da Saúde, no Brasil, o padrão de sazonalidade da gripe varia de acordo com a região analisada, sendo melhor observada naquelas que possuem estações climáticas bem definidas. Porém existe uma maior frequência nos meses mais frios (Ministério da Saúde, 2012).

Uma maneira simples de incluir tal sazonalidade no modelo SIR consiste em substituir a taxa de infecção fixa $(\beta)$ por uma taxa de infecção variante no tempo. Para esse propósito, adotou-se aqui uma função $\beta(t)$ dada por:

$$
\beta(t)=\left(\frac{\beta_{0}+\beta_{1}}{2}\right)-\left(\frac{\beta_{1}-\beta_{0}}{2}\right) \cos (\omega t),
$$

em que $\beta_{0}$ e $\beta_{1}$ são os valores mínimos e máximos respectivamente e $\omega=2 \pi / t_{f}$ é a frequência angular fixada. A Figura 1 mostra a função $\beta(t)$ para $t_{f}=100, \beta_{0}=0.1$ e $\beta_{1}=1$.

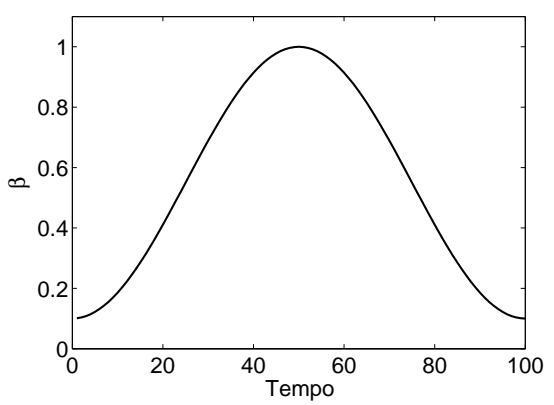

Figura 1: Taxa de infecção sazonal.

Vale ressaltar que para o caso da gripe, o modelo aplicado neste trabalho representa somente o contágio por um vírus específico, sem levar em conta possíveis mutações ao longo do período de tempo considerado.

\subsection{Politica de controle}

No intuito de reduzir a disseminação da moléstia na população este trabalho considera que seja adotada uma campanha para o uso de métodos de barreira. Tal método pode ser aplicado na sociedade através de campanhas de divulgação de bons hábitos de higiene e distribuição de máscaras, entre outras providências. Sua representação no modelo é dada pelo uso do controle visando à redução da taxa de infecção associada a $\beta(t)$.

Tendo em vista que a campanha requer um determinado tempo para que a sociedade assimile e pratique estes novos hábitos, faz-se necessário representar esta demora no modelo. Com esse intuito, o modelo SIR foi estendido com um estado 
adicional $C(t)$ na forma:

$$
\begin{aligned}
& d S(t) / d t=\mu N-\mu S(t)-\beta(t) \frac{I(t) S(t)}{N} C(t) \\
& d I(t) / d t=\beta(t) \frac{I(t) S(t)}{N} C(t)-\gamma I(t)-\mu I(t) \\
& d R(t) / d t=\gamma I(t)-\mu R(t) \\
& d C(t) / d t=(-C(t)+(1-u(t))) / \tau
\end{aligned}
$$

sendo $C(t)$ uma variável associada ao efeito da campanha na modificação dos hábitos da população e $u(t)$ a taxa temporal de aplicação de recursos na campanha (normalizada entre 0 e 1 ).

O problema de controle ótimo aqui considerado consiste em minimizar a função custo dada por:

$$
J(u)=\int_{t_{0}}^{t_{f}} I(t) d t,
$$

sujeito às Equações (4), (5) e à restrição isoperimétrica

$$
\int_{t_{0}}^{t_{f}} u(t) d t \leq h
$$

em que

$$
0 \leq u(t) \leq 1, \quad \forall t\left[0, t_{f}\right] .
$$

A restrição (7) impõe um limitante superior $\mathrm{h}$ para o total de recursos a serem empregados na campanha.

\subsection{Busca pelo controle ótimo}

Segundo (Morton and Wickwire, 1974) quando a função custo for linear em $u$, e as parcelas envolvendo $\mathrm{u}$ forem lineares nas equações do modelo, a equação de Bellman da programação dinâmica (e o Hamiltoniano na formulação do Princípio do Mínimo de Pontryagin) também irá conter $u$ linear. Deste modo, o controle ótimo, se ele existir, será do tipo "bang-bang" (Nepomuceno, 2005). No que segue, será apresentada uma demonstração dessa propriedade para o modelo específico empregado neste trabalho.

Dado o sistema

$$
\begin{aligned}
& d S(t) / d t=\mu N-\mu S(t)-\beta(t) \frac{I(t) S(t)}{N} C(t) \\
& d I(t) / d t=\beta(t) \frac{I(t) S(t)}{N} C(t)-\gamma I(t)-\mu I(t) \\
& d R(t) / d t=\gamma I(t)-\mu R(t) \\
& d C(t) / d t=(-C(t)+(1-u(t))) / \tau \\
& d D(t) / d t=u(t)
\end{aligned}
$$

em que $D(t)$ está relacionado à restrição isoperimétrica, deseja-se minimizar o custo (6).

O Hamiltoniano pode ser escrito por

$$
\begin{gathered}
H=I(t)+\lambda_{1}\left[\mu N-\mu S(t)-\beta(t) \frac{S(t) I(t)}{N} C(t)\right] \\
+\lambda_{2}\left[\beta(t) \frac{S(t) I(t)}{N} C(t)-\gamma I(t)-\mu I(t)\right] \\
+\lambda_{3}[\gamma I(t)-\mu R(t)] \\
+\lambda_{4}[(-C(t)+(1-u(t))) / \tau] \\
+\lambda_{5}[u(t)]
\end{gathered}
$$

Com isso, as equações de coestado, dadas por $\dot{\lambda}=$ $-H_{x}$, podem ser escritas como:

$$
\begin{aligned}
\dot{\lambda_{1}}=-H_{S}= & {\left[\mu+\beta(t) \frac{I(t) C(t)}{N}\right] \lambda_{1} } \\
& -\left[\beta(t) \frac{I(t) C(t)}{N}\right] \lambda_{2} \\
\dot{\lambda_{2}}=-H_{I}= & {\left[\mu-\beta(t) \frac{S(t) C(t)}{N}\right] \lambda_{2} } \\
& +\left[\beta(t) \frac{S(t) C(t)}{N}\right] \lambda_{1} \\
& +\left(\lambda_{2}-\lambda_{3}\right) \gamma \\
\dot{\lambda_{3}}=-H_{R}= & \mu \lambda_{3} \\
\dot{\lambda_{4}}=-H_{C}= & \frac{\lambda_{4}}{\tau}+\beta(t) \frac{S(t) I(t)}{N}\left[\lambda_{1}-\lambda_{2}\right] \\
\dot{\lambda_{5}}=-H_{D}= & 0
\end{aligned}
$$

obtendo-se

$$
\lambda_{5}=c_{5}
$$

em que $c_{5}$ é uma constante.

Isolando os termos dependentes do controle $u(t)$ da Equação (10), tem-se

$$
\left[-\frac{\lambda_{4}}{\tau}+\lambda_{5}\right] u(t)
$$

substituindo (12) em (13), segundo o Princípio do Mínimo de Pontryagin, pode-se concluir que:

$$
u^{*}(t)=\left\{\begin{array}{lll}
0, & \text { se } & -\frac{\lambda_{4}}{\tau}+c_{5}>c_{5} \\
1, & \text { se } & -\frac{\lambda_{4}}{\tau}+c_{5}<c_{5}
\end{array}\right.
$$

Em outras palavras, se o coeficiente que multiplica $u(t)$ na Equação (13) for positivo $u(t)$ deve ser mínimo, e se for negativo deve ser máximo. Demonstra-se assim que o controle será do tipo "bang-bang".

\section{Material e Métodos}

É sabido que o controle ótimo é do tipo "bangbang", porém não é possível afirmar quantos pulsos o controle apresenta e nem o melhor momento para aplicá-los. Para tentar contornar este problema, este trabalho propõe uma busca em grade por um controle do tipo "bang".

Neste trabalho, o modelo SIR descrito na Seção 2 foi simulado baseando-se no método numérico de Dormand-Prince (Dormand and Prince, 1980). Tal implementação foi configurada com os seguintes parâmetros normalizados em termos de unidades de tempo, indivíduos e recursos (Galvão Filho et al., 2011):

$$
\begin{aligned}
t_{0} & =0 \\
t_{f} & =100 \\
\mu & =0.005 \\
\gamma & =1 / 15 \\
\beta_{0} & =0.1 \\
\beta_{1} & =1 \\
\omega & =2 \pi / t_{f} \\
h & =50
\end{aligned}
$$


Para analisar o efeito do tempo envolvido na assimilação da campanha, a constante de tempo $\tau$ foi variada de 0 a 40 . Para cada configuração de $\tau$ foi realizado uma busca pela melhor alocação do controle. O pulso teve duração de 50 unidades de tempo, de modo a respeitar a restrição isoperimétrica. Desta forma foi considerado inicialmente apenas um pulso, buscando assim o tempo $t_{1}^{*}$ no qual será dado o início. O mesmo cenário foi simulado para dois pulsos, ou seja dividindo os recursos em duas partes de 25 unidades e buscando a melhor alocação para ambos no horizonte de tempo. O tempo de alocação ótimo para os pulsos foram chamados de $t_{1}^{*}$ e $t_{2}^{*}$, representando assim o menor custo obtido iniciando o primeiro e o segundo pulso respectivamente. A Figura 2 mostra um exemplo para (a) um pulso com $t_{1}=20 \mathrm{e}$ (b) $\operatorname{com} t_{1}=15$ e $t_{2}=60$
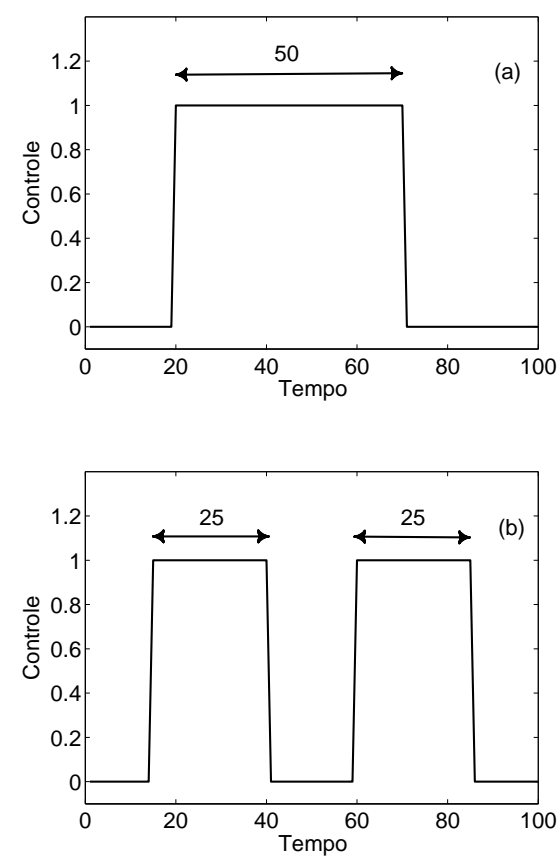

Figura 2: Exemplos de controle com (a) um e (b) dois pulsos.

\section{Resultados}

A Figura 3 apresenta os resultados da simulação utilizando os parâmetros citados na Seção $3 \mathrm{com}$ $\tau=0$ e $\tau=10$. A Figura 3b mostra que o controle ótimo foi alocado aproximadamente no centro do horizonte de tempo, ou seja o mais próximo possível do ápice da sazonalidade. Assim é possível observar na Figura 3a que o método de barreira é instantaneamente aplicado, e consequentemente neste intervalo de tempo o contágio é eliminado. Nas regiões em que o controle não está atuando a infecção se faz presente, porém no momento mais brando da sazonalidade. A Figura 3c mostra o resultado da simulação para $\tau=10$ considerando
Tabela 1: Resultado da otimização com um e dois pulsos para $\tau=[0,5,10,15,20]$

\begin{tabular}{|l|l|l|l|l|}
\hline & \multicolumn{2}{|c|}{1 pulso } & \multicolumn{2}{|c|}{2 pulsos } \\
\hline$\tau=0$ & $t_{1}^{*}=19$ & $J^{*}=11463$ & $t_{1}^{*}=19$ & $J^{*}=9995$ \\
& & & $t_{2}^{*}=47$ & \\
\hline$\tau=5$ & $t_{1}^{*}=15$ & $J^{*}=11927$ & $t_{1}^{*}=15$ & $J^{*}=11927$ \\
& & & $t_{2}^{*}=40$ & \\
\hline$\tau=10$ & $t_{1}^{*}=12$ & $J^{*}=13038$ & $t_{1}^{*}=9$ & $J^{*}=12054$ \\
& & & $t_{2}^{*}=37$ & \\
\hline$\tau=15$ & $t_{1}^{*}=9$ & $J^{*}=14170$ & $t_{1}^{*}=4$ & $J^{*}=13375$ \\
& & & $t_{2}^{*}=33$ & \\
\hline$\tau=20$ & $t_{1}^{*}=6$ & $J^{*}=14983$ & $t_{1}^{*}=6$ & $J^{*}=14541$ \\
& & & $t_{2}^{*}=34$ & \\
\hline
\end{tabular}

o controle apresentado na Figura 3b. Observa-se um aumento significativo dos indivíduos recuperados e consequentemente a redução dos indivíduos suscetíveis, o que significa que houve um maior número de contágios. Tal aumento se deve ao fato de que a constante de tempo foi adicionada e assim a campanha só será efetiva posteriormente. Portanto a população ficará exposta a uma taxa de infecção maior. Quando o controle ótimo levando em conta a constante de tempo é aplicado verificase uma antecipação da ação de controle em relação ao pico da sazonalidade. Uma vez que a constante de tempo irá atrasar a eficácia do método de barreira é sensato adiantar a ação de controle para que o método esteja vigorando no pico da sazonalidade. As Figuras 3d e 3e corroboram tal análise. De modo similar ao primeiro caso, é possível observar uma redução no número de indivíduos infectados no decorrer do horizonte de tempo.

A Tabela 1 mostra o tempo inicial e o custo obtido com o controle contendo um e dois pulsos. Verifica-se que os tempos encontrados são muito próximos comparando os dois cenários. À medida que $\tau$ aumenta, os tempos diminuem de maneira similar. É possível observar também que para todos os valores de $\tau$ analisados, na estratégia de dois pulsos, o menor custo foi obtido agrupando os dois pulsos (formando assim um pulso só) ou alocando-os próximo um do outro. Isso significa que para a dinâmica sazonal proposta neste trabalho, as duas estratégias resultam em ações de controle similares. Assim, a redução do custo obtida com dois pulsos não foi considerada significativa. Do ponto de vista prático utilizar os dois pulsos seria o mesmo que aplicar a campanha em um período contínuo com uma pequena interrupção. Isso dificulta a logística da campanha, pois seria necessário interromper a campanha no meio por um curto período de tempo. Logo, por ser mais simples, a estratégia utilizando apenas um pulso será recomendada.

A Figura 4 apresenta os valores de $t_{1}^{*}$ juntamente com as curvas $J$ com seus respectivos $t_{1}$ para valores de $\tau=[0,1, \ldots, 40]$. 

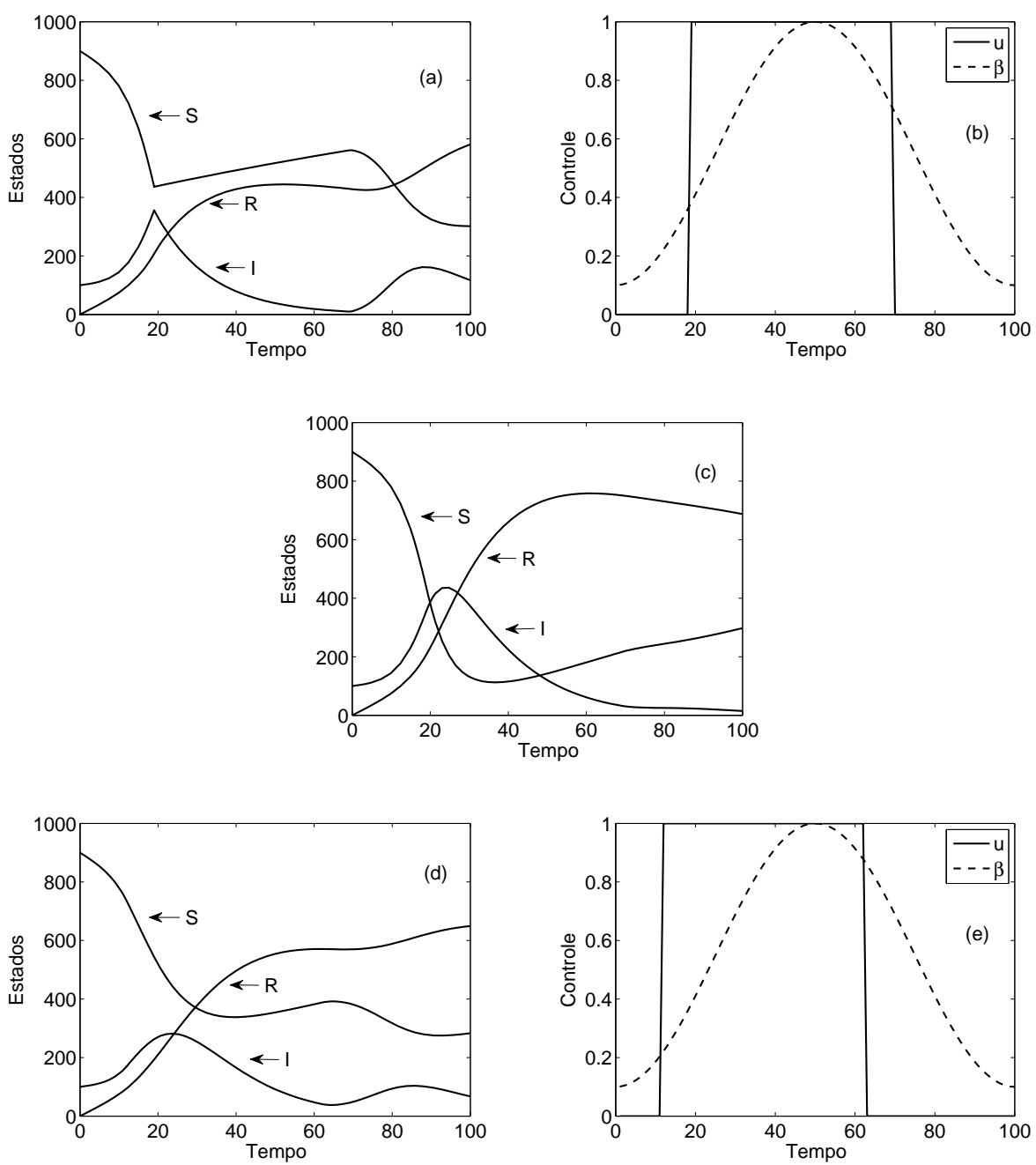

Figura 3: Resultado de três simulações contendo (a) os estados e (b) o controle ótimo para $\tau=0$, (c) os estados para $\tau=10$ utilizando o controle obtido em (b) e (d) estados e (e) o controle para $\tau=10$.

Pode-se observar na Figura 4a que à medida que $\tau$ aumenta, $t_{1}$ tende a diminuir. Tal fenômeno pode ser associado ao fato de que quanto maior o $\tau$ mais lenta será a dinâmica da assimilação da campanha e consequentemente o controle deverá ser aplicado mais precocemente. A Figura $4 \mathrm{~b}$ corrobora a análise anterior, mostrando que, com o aumento de $\tau, t_{1}^{*}$ diminui e $J^{*}$ tende a aumentar. Vale salientar que para valores de $\tau>20$ aproximadamente, as curvas de $J$ mostram uma pequena sensibilidade para variações de $t_{1}$. Este comportamento ilustra que quando a constante de tempo $\tau$ é muito grande, a campanha acaba não surtindo efeitos, e assim a escolha de $t_{1}$ não irá reduzir o custo expressivamente.

\section{Conclusões}

Este trabalho propôs o uso do SIR para alocação temporal de um controle ótimo para a dinâmica da influenza sazonal utilizando métodos de barreira e com recursos limitados. Verificou-se que o controle ótimo escolhido alocou os recursos na re- gião do horizonte de tempo em que o contágio era mais intenso, quando aplicado instantaneamente. Quando a campanha requer um tempo para que se torne efetiva, observou-se que as menores quantidades de indivíduos infectados no decorrer da simulação foram apresentadas adiantando a ação de controle.

O modelo SIR empregado neste trabalho foi relativamente simples, de modo a verificar o comportamento da dinâmica proposta sob uma campanha baseada em métodos de barreira. Em trabalhos futuros, pretende-se explorar outras formas de controle da influenza, como a aplicação da vacina na população. Espera-se que nesse caso a redução do número de indivíduos infectados seja ainda mais expressiva, devido à imunização contra a doença.

\section{Agradecimentos}

Os autores agradecem à Capes e ao $\mathrm{CNPq}$ pelos auxílios concedidos. 

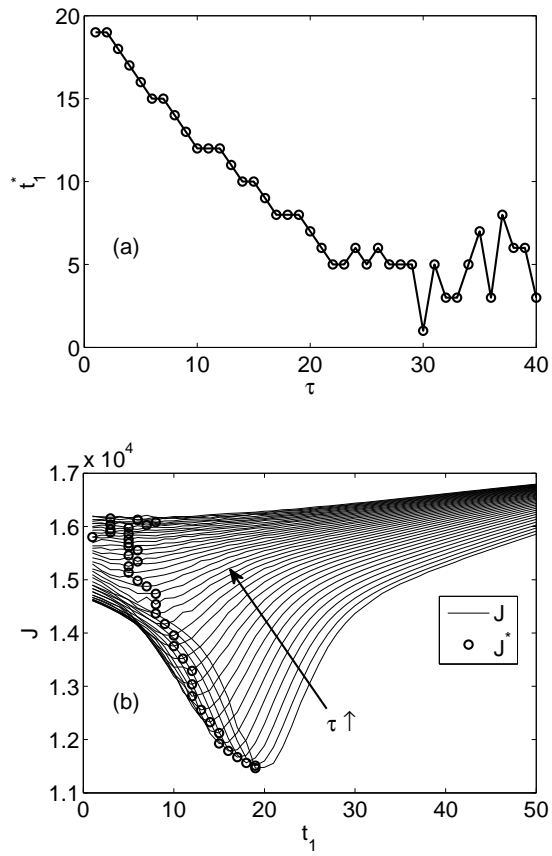

Figura 4: Resultados da otimização empregando um pulso, para diferentes valores de $\tau$.

\section{Referências}

Anderson, R. M. and May, R. M. (1992). Infectious diseases of humans: Dinamics and control, Oxford University Press.

Behncke, H. (2000). Optimal control of deterministic epidemics, 21: 269-285.

Dormand, J. R. and Prince, P. J. (1980). A family of embedded runge-kutta formulae, $6(1)$ : 1926 .

Filho, N. A. and Rouquayrol, M. Z. (2006). Introdução à epidemiologia, Guanabara Koogan.

Galvão Filho, A. R., Arruda, F. D. B., Galvão, R. K. H. and Yoneyama, T. (2011). Programação paralela CUDA para simulação de modelos epidemiológicos baseados em indivíduos, Em: Anais do X Simpósio Brasileiro de Automação Inteligente, São João del-Rei, $M G$.

Giancotti, K. H. O., de Assis Dias, F., M.Texeira, W. W., Nepomuceno, E. G. and Kurcbart, S. M. (2010). Análise da estrutura do MBI: sensibilidade da taxa de infecção e da população, Em: Anais do XVIII Congresso Brasileiro de Automática, Bonito, MS.

Grenfell, B. T., Bjonstad, O. N. and Finkenstadt, B. F. (2002). Dinamics of measles epidemic: scaling noise, determination, and predictability with the TSIR model, 72: 185-202.
Hansen, E. and Day, T. (2011). Optimal control of epidemics with limited resources, 62: 423451.

Kermack, W. and Mckendrick, A. (1927). A contribution to the mathematical theory of epidemics, 115: 700 - 721 .

Lu, Z., Chi, X. and Chen, L. (2002). The effect of constant and pulse vaccination on SIR epidemic model with horizontal and vertical transmission, 36: 1039 - 1057.

Ministério da Saúde (2012). Boletim informativo de influenza, http://portalsaude.saude. gov.br/portalsaude/noticia/6651/785/ boletim-informativo-de-influenza: -semana-epidemiologica-32.html. [Online; acessado 1-maio-2013].

Morton, R. and Wickwire, K. H. (1974). On the optimal control of a deterministic epidemic, 6(4): 622-635.

Nepomuceno, E. G. (2005). Dinâmica, modelagem e controle de epidemias, $\mathrm{PhD}$ thesis, Universidade Federal de Minas Gerais, Belo Horizonte, MG.

Prosper, O. and Saucedo, O. (2011). Modeling control strategies for concurrent epidemics of seasonal and pandemic H1N1 influenza, 8(1): 141-170.

World Health Organization (2009). Influenza (seasonal): Fact sheet $\mathrm{n}^{\mathrm{O}} 211$, http: //www . who . int/mediacentre/factsheets/fs211/en/. [Online; acessado 13-maio-2013].

Yoshida, N. and Hara, T. (2007). Global stability of a delayed sir epidemic model with density dependent birth and death rates, 201: 339347. 